\title{
Aa. Vv., Etudes rabelaisiennes
}

\section{Filippo Fonio}

\section{(2) OpenEdition}

\section{Journals}

\section{Edizione digitale}

URL: https://journals.openedition.org/studifrancesi/39092

DOI: 10.4000/studifrancesi.39092

ISSN: 2421-5856

\section{Editore}

Rosenberg \& Sellier

\section{Edizione cartacea}

Data di pubblicazione: 1 décembre 2004

Paginazione: 346-347

ISSN: 0039-2944

\section{Notizia bibliografica digitale}

Filippo Fonio, «Aa. Vv., Etudes rabelaisiennes», Studi Francesi [Online], 143 (XLVIII | II) | 2004, online dal 30 novembre 2015, consultato il 19 mai 2021. URL: http://journals.openedition.org/studifrancesi/ 39092 ; DOI: https://doi.org/10.4000/studifrancesi.39092

Questo documento è stato generato automaticamente il 19 mai 2021.

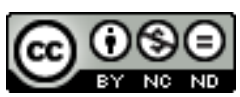

Studi Francesi è distribuita con Licenza Creative Commons Attribuzione - Non commerciale - Non opere derivate 4.0 Internazionale. 


\title{
Aa. Vv., Etudes rabelaisiennes
}

\author{
Filippo Fonio
}

\section{NOTIZIA}

ETUDES RABELAISIENNES, Tome XLII, Genève, Droz ("Travaux d'Humanisme et Renaissance", CCCLXXIX), 2003, pp. 143.

1 Il volume XLII delle Études rabelaisiennes consta di cinque contributi e di un indice dei rimandi al corpus rabelaisiano, relativi ai primi quaranta volumi della pubblicazione (DAMIEN DARD, Index des ceuvres de Rabelais dans les quarante premiers volumes des Études rabelaisiennes, pp. 125-140). L'indice è redatto sulla base dei singoli capitoli o, nel caso di studi relativi a episodi più estesi, di gruppi di capitoli, secondo la ripartizione adottata da Mireille Huchon nella recente edizione critica (FRANçoIS RABELAIS, Euvres complètes, Paris, Gallimard, "Bibliothèque de la Pléiade", 1994).

Il primo studio qui raccolto è incentrato sulla retorica e la stilistica rabelaisiane: si tratta di FRANçOIS CORNILLIAT, On sound effects in Rabelais (part II), pp. 7-55. La prima parte del saggio, relativa all'uso dell'allitterazione e del similiter desinens, si trova in Ètudes rabelaisiennes, XXXIX, Genève, Droz, 2000, pp. 137-167. Il saggio di Cornilliat è un contributo alla teoria dell'imitazione in Rabelais, partendo in questo caso dai rhétoriqueurs per osservare come l'impiego dei medesimi procedimenti stilistici, in particolare la paronomasia, possa convogliare effetti diversi al variare del contesto, o nel momento in cui viene introdotto un procedimento parodico. Lo studio in questione verte sull'art of punning rabelaisiana (su cui cfr. in part. FRANÇOIS RIGOLOT, Les langages de Rabelais, Genève, Droz, 1996, oltre agli studi di Guy Demerson), prendendo in esame i luoghi dei romanzi dove più liberamente si esplica l'inventività verbale di Alcofribas Nasier e di François Rabelais narratori, oppure dei personaggi: la copia, il blason, gli elogi spesso paradossali, la satira nei confronti della philautia, le isotopie riconducibili all'area semantica del debito, il discorso del buffone come rilettura della moria erasmiana, le dinamiche dei dialoghi fra i personaggi. Proprio nei rhétoriqueurs sarebbe riscontrabile in nuce lo scarto verso un'autonomia del significante con finalità 
espressive, la de-referenzializzazione del linguaggio verso la pura phoné, presente in alcuni passi dell'opera di Rabelais: "rhétoriqueur practice also shows that there can be a build-up effect from object-saturated to sound-saturated lists - from naming to singing, from referential to poetic copia", p. 45. Diversi sono i prelievi testuali, in particolare da Jean Lemaire de Belges, Illustrations de Gaule et Singularitez de Troye, e André de la Vigne, Voyage de Naples, a confronto con Rabelais. L'accostamento evidenzia tanto la modalità di impiego delle fonti all'interno dell'opera pantagruelica, quanto la maniera in cui Rabelais smaschera alcuni procedimenti dei rhétoriqueurs, contribuendo all'acquisizione di una maggiore consapevolezza degli artifici presenti nelle pratiche linguistiche e, suggerendo l'inscindibilità della componente artificiosa del linguaggio rispetto a quella referenziale, a mostrare come essa possa essere fatta funzionare a fini espressivi. "The twist - whose Evangelical flavour is unmistakable - is that language, when aware of its own emptiness, proves actually less empty, and less dangerous, than when it persuades itself of its motivation", p. 50.

3 JAMES HELGESON si occupa di un problema semiotico e filosofico al centro del dibattito novecentesco: "whether meaning implies an internal intentional on the part of a speaking subject", p. 75, analizzando la posizione di Rabelais medico, versato in diritto ed esegesi biblica, conoscitore del neoplatonismo, riguardo all'intenzionalità del significato ("Ce que j'entends par ces symboles pythagoricques": Rabelais on meaning and intention, pp. 75-100). Circa dieci sono le occorrenze di entendre (intention) nei romanzi di Rabelais, oscillanti fra le accezioni understand, intend, mean (queste ultime con una sfumatura intenzionale). Nel rapporto fra scriptum e voluntas entrerebbero in gioco varie componenti, fra cui l'intenzionalità appunto, ma anche l'ispirazione, che ad essa in certo modo si contrappone, la pratica allegorica in fase di scrittura (volontà autoriale) e di interpretazione (la preminenza accordata all'esegeta e la nozione di comunità interpretativa), la trama di un subtesto che l'autore definisce la "scrittura segreta" di Rabelais.

4 Sulla questione delle fonti intesa in senso lato vertono gli studi di FRÉDÉRIC TINGUELY, L'alter sensus des turqueries de Panurge, pp. 57-73, e di FRANÇOIS ROUGET, Rabelais lecteur de Castiglione et de Machiavel à Thélème (Gargantua, chap. 52-57), pp. 101-116.

5 Tinguely, anche sulla scorta delle analisi di Gérard Defaux, individua la componente odissaica del personaggio di Panurge fin dalla sua entrata in scena (Pantagruel, IX), vedendo delle analogie fra le menzogne iperboliche di cui Panurge infarcisce il resoconto delle avventure in Turchia e il colloquio fra Ulisse ed Eumeo. La fonte principale del discorso sui Turchi svolto nel Pantagruel sarebbe l'erasmiana Utilissima consultatio de bello Turcis inferendo (1530), in particolare per l'ambiguità che sostanzia l'atteggiamento nei confronti dell'altro: "Comme celle d'Erasme, la représentation de l'altérité musulmane proposée par Rabelais témoignerait en fin de compte des limites du discours humaniste dès lors qu'il se trouve aux prises avec la différence", p. 60. La tematica evangelica, e forse un attacco ad personam rivolto a un teologo della Sorbona sarebbero celati nell'episodio dei Turchi, nel quale dunque due idee di crociata e di guerra santa si sovrapporrebbero, contribuendo alla composizione di un passo di satira militante.

6 Rouget individua in Castiglione e Machiavelli, nel Cortegiano e nei Capitoli per una compagnia di piacere i due ipotesti in base ai quali Rabelais costruisce la celebre descrizione di Thélème e dei Telemiti. Thélème è un'utopia umanista, un'anti-abbazia non in quanto rovesciamento dell'ideale monastico, ma perché ad esso sostituisce un 
ideale cortese-cortigiano. Molti i riscontri testuali nei confronti del Cortegiano, ma lo spirito e il tono dell'episodio di Thélème si avvicinerebbero alla satira della società di corte nelle forme messe in atto da Machiavelli nell'opera citata. Un simile accostamento permette fra l'altro di rendere ragione dell'apparente isolamento dell'episodio all'interno della tonalità del Pantagruel. Sono presenti il testo dei Capitoli di Machiavelli e la traduzione francese.

7 Una presa di posizione da parte di Rabelais nell'ambito della polemica sulla nazionalità del primo libraio romano, Uldaricus Han, è messa in luce da STÉPHAN GEONGET (Rabelais, son coq et ses gelines: la basse cour d'Ulrich Galet, pp. 117-124). Il nome di Ulrich Gallet, "maistre des requestes" di Grandgousier (Gargantua, XXX-XXXI) sarebbe la risposta gallicana di Rabelais a Jacques Wimpheling, che nella Rerum Germanicarum epitome (1505) fra le lodi iperboliche rivolte alla Germania avrebbe attribuito nazionalità tedesca al primo libraio romano. La posizione di Rabelais, che come Guillaume Du Bellay fra gli altri risponde all'epitome tedesca, si basa sullo slittamento paraetimologico Uldaricus Hahn - Uldaricus Gallus - Ulrich le Français. 